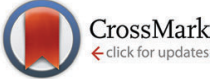

Cite this: Phys. Chem. Chem. Phys., 2016, 18, 2924

Received 19th November 2015, Accepted 22nd December 2015

DOI: $10.1039 / \mathrm{c} 5 \mathrm{cp} 07116 \mathrm{j}$

www.rsc.org/pccp

\title{
Charge distribution and Fermi level in bimetallic nanoparticles $\dagger$
}

\author{
Nico Holmberg, ${ }^{a}$ Kari Laasonen ${ }^{\star a}$ and Pekka Peljo*b
}

\begin{abstract}
Upon metal-metal contact, a transfer of electrons will occur between the metals until the Fermi levels in both phases are equal, resulting in a net charge difference across the metal-metal interface. Here, we have examined this contact electrification in bimetallic model systems composed of mixed Au-Ag nanoparticles containing ca. 600 atoms using density functional theory calculations. We present a new model to explain this charge transfer by considering the bimetallic system as a nanocapacitor with a potential difference equal to the work function difference, and with most of the transferred charge located directly at the contact interface. Identical results were obtained by considering surface contacts as well as by employing a continuum model, confirming that this model is general and can be applied to any multimetallic structure regardless of geometry or size (going from nano- to macroscale). Furthermore, the equilibrium Fermi level was found to be strongly dependent on the surface coverage of different metals, enabling the construction of scaling relations. We believe that the charge transfer due to Fermi level equilibration has a profound effect on the catalytic, electrocatalytic and other properties of bimetallic particles. Additionally, bimetallic nanoparticles are expected to have very interesting self-assembly for large superstructures due to the surface charge anisotropy between the two metals.
\end{abstract}

\section{Introduction}

In recent years, bimetallic nanoparticles have received increasing attention due to their promising electrocatalytic, ${ }^{1-3}$ catalytic, ${ }^{4-8}$ magnetic, ${ }^{4,9,10}$ and optical properties. ${ }^{4,11}$ The interaction of nanoparticles with their environment can, sometimes drastically, shift the Fermi level of electrons in the nanoparticles, influencing their chemical and electrochemical properties, as highlighted in a recent review. ${ }^{12}$ Another effect largely neglected in the literature is the effect of contact electrification on shifting the Fermi level of electrons in bi- or multimetallic structures. It is well known that contact charging takes place when two materials with a different Fermi level of electrons are brought together. Once in contact, electrons will flow from the material with higher Fermi level into the other, until equilibrium is reached, and the Fermi levels have equalized. This charge transfer has been observed for example by density functional theory calculations. ${ }^{13-19}$ However, the effects of this charge transfer have mostly been neglected thus far in the

\footnotetext{
${ }^{a}$ COMP Centre of Excellence in Computational Nanoscience, Department of Chemistry, Aalto University, P.O. Box 16100, FI-00076 Aalto, Finland. E-mail: kari.laasonen@aalto.fi

${ }^{b}$ Laboratoire d'Electrochimie Physique et Analytique,

École Polytechnique Fédérale de Lausanne, EPFL Valais Wallis, Rue de l'Industrie 17, Case Postale 440,CH-1951 Sion, Switzerland. E-mail: pekka.peljo@epfl.ch $\dagger$ Electronic supplementary information (ESI) available: Details of the computational methods, additional results as tables and figures, more comprehensive theory of contact electrification. See DOI: 10.1039/c5cp07116j
}

literature, with the exception of studies focused on the stabilizing effects of charge transfer for certain structures. ${ }^{4,15}$ The aim of this article is to clarify what will be the position of the Fermi level after contact charging, to understand how much charge is transferred, and where this charge is located. This charge transfer can have significant effects on, e.g., electrocatalytic, catalytic and optical properties. For example, bimetallic nanosystems are reported to have a markedly different absorbance of light than systems constructed only from a single element, ${ }^{20,21}$ while core-shell and Janus particles have highly different optical responses. ${ }^{22}$

Based on extensive density functional theory simulations, the equilibrium Fermi level of a bimetallic system was found to be strongly dependent on the surface coverage of different metals in the structure. The results indicate that bimetallic nanoparticles can actually be considered as attocapacitors, with most of the transferred charge located at the contact interface and only a small fraction on the outer surface. Furthermore, the current results also predict that charge transfer in bimetallic Janus particles will result in the formation of electrical dipoles. Like Janus particles of a single metal covered with different surface groups, these bimetallic Janus particles are expected to have very interesting self-assembly for formation of superstructures, which could not be formed from homogeneous particles. ${ }^{23,24}$ As nanoparticles themselves exhibit very interesting self-assembly behavior, ${ }^{25,26}$ this self-polarization also results in additional possibilities to use these types of particles for hierarchical assembly, one of the most appealing targets in nanoscience. ${ }^{27}$ 
The idea behind hierarchical assembly is to use nanoparticles and other building blocks to produce materials with tailored properties. As the self-assembly is typically driven by electrostatic, van der Waals, hydrophobic, and other interactions at the nanoscale, ${ }^{28}$ the formation of electrostatic dipoles due to contact charging gives one more tool to induce this self-assembly. As different synthesis methods for bimetallic Janus particles and crystals have been recently developed, ${ }^{5,29-31}$ behavior of these particles in an electric field, their self-assembly and superstructure formation will be of considerable further interest.

\section{Results and discussion}

\subsection{Fermi level equilibration in bimetallic nanoparticles}

Fermi level equilibration and the associated charge transfer was investigated in bimetallic systems by performing density functional theory (DFT) calculations at the PBE/GGA ${ }^{34}$ level of theory, as detailed in the ESI. $\dagger$ Specifically, we have chosen a model system composed of silver and gold because of the matching sizes of the atoms and the availability of reliable work function data. The contact electrification between silver and gold was studied with bimetallic nanoparticles, and the results were corroborated by comparison with calculations on bimetallic surfaces. As the aim of the paper was to study contact electrification, stability of the selected structures was not considered.

Charge transfer at the $\mathrm{Ag} / \mathrm{Au}$ interface was investigated using a total of 17 icosahedral nanoparticles with a fixed size of 561 atoms, as illustrated in Fig. 1 and Fig. S1 in the ESI. $\dagger$ We have investigated both the effects of nanoparticle shell $\mathrm{Ag} / \mathrm{Au}$ composition at a fixed atomic configuration $\mathrm{Ag}_{252} \mathrm{Au}_{309}$ (Fig. 1), as well as the effects of atomic configuration $\mathrm{Ag}_{x} \mathrm{Au}_{561-x}$ with three shell compositions (Fig. S1, ESI $\dagger$ ). All nanoparticles were placed in vacuum inside a cubic simulation cell with $6 \mathrm{~nm}$ edge lengths. Prior to analysis, the nanoparticles were relaxed until the maximum force decreased below $0.023 \mathrm{eV}^{-1}$.

Calculated nanoparticle equilibrium Fermi levels are shown in Fig. 2. For bimetallic nanoparticles, the Fermi level is linearly dependent on the surface atom $\mathrm{Ag} / \mathrm{Au}$ ratio decreasing from $-4.25 \mathrm{eV}$ with a pure silver particle to $-5.11 \mathrm{eV}$ for a

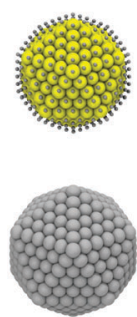

$0 \%$
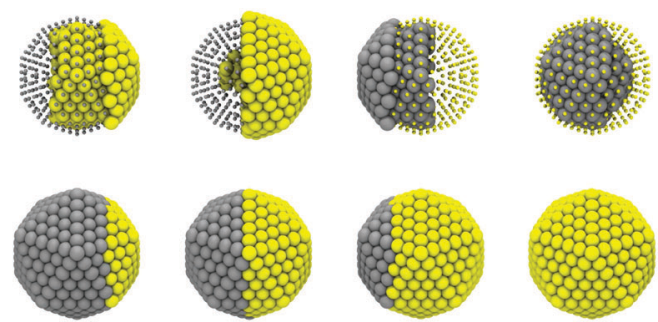

$50 \%$
$75 \%$

$100 \%$
Fig. 1 Optimized structures of nanoparticles with fixed atomic configuration $\mathrm{Ag}_{252} \mathrm{Au}_{309}$. Gold atoms are shown in yellow and silver atoms in gray. The fraction of Au in the shell layer is indicated. The particle with $50 \%$ $\mathrm{Au}$ and $\mathrm{Ag}$ in the shell layer is a Janus particle, whereas the particles with only a single metal in the shell are core-shell particles. The snapshots have been generated using VMD. ${ }^{32,33}$

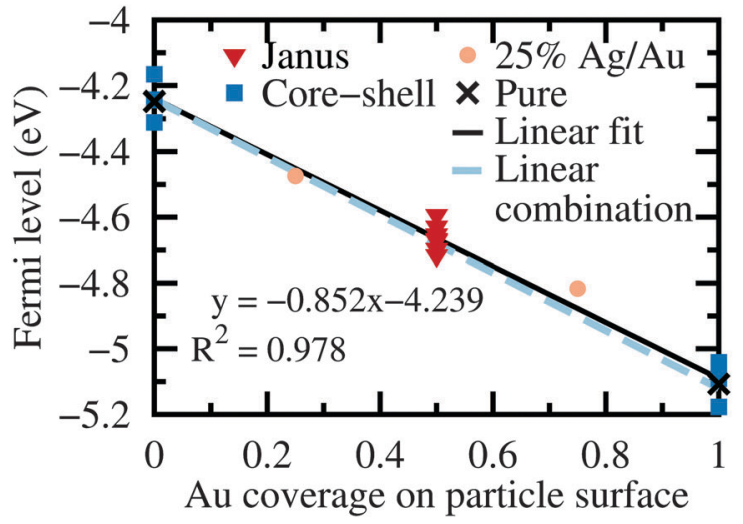

Fig. 2 Computational Fermi levels of the investigated pure and bimetallic nanoparticles with different composition as a function of Au surface coverage. The solid line is a linear fit to all data points with fit indicated, while the dashed line is the shell composition weighted average calculated according to eqn (1).

pure gold particle. In fact, the Fermi level of these systems, $E_{\mathrm{F}}$, can simply be estimated by

$$
E_{\mathrm{F}, \mathrm{Au}-\mathrm{Ag}}=x_{\mathrm{S}, \mathrm{Au}} E_{\mathrm{F}, \mathrm{Au}}+x_{\mathrm{S}, \mathrm{Ag}} E_{\mathrm{F}, \mathrm{Ag}}
$$

where $x_{\mathrm{S}, \mathrm{i}}$ is the surface area ratio of $\mathrm{i}$. This relationship coincides perfectly with the linear fit to DFT evaluated values, and is already well known. ${ }^{35,36}$ In the case of polycrystalline surfaces, the average work function of the surface is the weighted average of the work functions of the individual crystallites, known as "patches",. ${ }^{35,36}$ For planar, cylindrical, and spherical surfaces, the average work function is the surface area weighted average of the work functions of the individual patches. ${ }^{35,36}$ For more complex geometries, the electrostatic interactions between oppositely charged patches has to be considered more carefully, as described by for example Sahni et $a .^{35}$ and Baldereschi et al. ${ }^{37}$ Same considerations apply for the surfaces covered with patches of different metals, but in this case Fermi level differences between the two metals can be much higher, on the order of eV.

The amount of charge transferred, on the other hand, depends strongly on the geometry of the system. For example, on the particles of same composition $\mathrm{Ag}_{252} \mathrm{Au}_{309}$ but different surface coverages, the total charge transferred in the case of a core-shell particle with Ag on the surface is ca. 12 electrons, with a minimum for the Janus particle and further increase towards the particle fully covered in gold. The data for all the considered particles is presented in the ESI, $\dagger$ see Table S1. To understand why the charge transfer is so strongly dependent on the geometry, we must first consider the theory of contact electrification.

Upon metal-metal contact, electrons can freely exchange between the two conductors. In most cases, the initial Fermi levels of the metals are not equal, so a net flow of electrons takes place from the material with the lower work function into the other until the Fermi levels are equal in both phases. This results in an outer potential $\psi$ difference between the two metals, which is directly proportional to the work function 
$\Phi$ difference between the two metals (for full discussion, see the ESI $\dagger$ )

$$
\psi^{\mathrm{II}}-\psi^{\mathrm{I}}=\frac{\Phi^{\mathrm{I}}-\Phi^{\mathrm{II}}}{e}
$$

If we consider that the two metal bodies of the nanoparticles form a capacitor, where both parts can be considered as an equipotential metal body separated by a very small distance, the observed charge transfer trends can reasonably well be explained with this relatively basic model. Capacitance can simply be considered as

$$
C=\frac{q}{V} \approx \frac{\varepsilon_{0} A}{d}
$$

where $q$ is the charge in each metal, $V=\psi^{\mathrm{II}}-\psi^{\mathrm{I}}$ is the potential difference, $\varepsilon_{0}$ is the permittivity of vacuum, $A$ is the contact area between the two metals, and $d$ is the distance. Here, we assume that the system can be approximated as a parallel plate capacitor. The interfacial area between the two metals was estimated by the solvent accessible surface area (SASA) method utilizing a probe of $0.8 \AA$ radius, ${ }^{32,38,39}$ as described in the ESI. $\dagger$ As the areas of Au and $\mathrm{Ag}$ at the interface are different, the average area was used, while the average distance between the centers of interfacial $\mathrm{Au}$ and $\mathrm{Ag}$ atoms was employed for the distance $d$. The capacitance of the system can then be evaluated using the calculated net charge transfer magnitudes and considering that the potential difference is the same as the contact potential difference, which according to eqn (2) is directly dependent on the work function difference of the two pure metals. In this case, the potential difference was calculated from the work functions of the pure metal nanoparticles. Even though there is a potential difference between the two metals, electrons can freely tunnel through the gap between the phases and these junctions offer very little resistance for the passage of current, since both phases are very close to each other. The computational capacitances (in aF) of the investigated nanoparticles are given as a function of contact area/contact distance $(A / d$; in $\AA$ ) in Fig. 3 .

Fig. 3 shows that the capacitance of the core-shell particles increases with growing core size, while the Janus particles all have relatively similar capacitances. The variation in the plot most probably arises from the difficulties in accurately estimating the interfacial area and the distance between $\mathrm{Ag} / \mathrm{Au}$ phases. Additionally, the system should not be described as a parallel plate capacitor, because borders of the metals form quite sharp edges especially in the particles with 25 and $75 \%$ gold on the surface. A more refined model for the capacitance should lead to more accurate results, but this simplified model is enough to quantitatively understand the variation in the amount of charge transferred for different nanoparticles. The slope of the linear fit in Fig. 3 should give the permittivity of the vacuum. However, as the SASA method estimates the surface area of the system at a distance of probe radius away from the probed atoms, the method overestimates the surface area. Furthermore, the average distance between the centers of interfacial $\mathrm{Ag}$ and $\mathrm{Au}$ atoms should be replaced by a distance distribution, so now the distances are also overestimated. Considering these limitations, eqn (3) shows a reasonable correlation between the computational capacitance

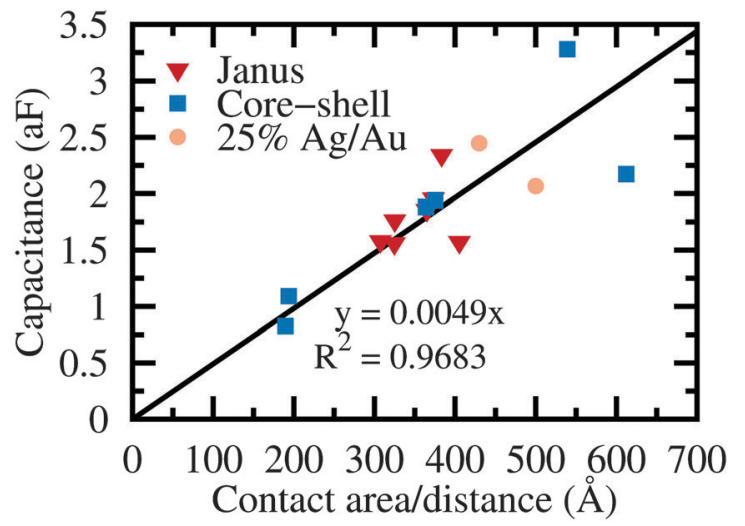

Fig. 3 Computational capacitances of different bimetallic nanoparticles as the function of contact area/contact distance, $A / d$.

and the geometry of the contact interface, quantitatively explaining the magnitude of charge transfer from $\mathrm{Ag}$ to $\mathrm{Au}$ in different bimetallic nanoparticles.

From an experimental perspective, this simple model can be justified, for example, by the observations of Harper ${ }^{40}$ who studied charge transfer between two spheres composed of different metals. First, the spheres were brought into contact to allow their Fermi levels to equilibrate, charging the metals. Subsequently, the spheres were again separated leaving a measurable residual charge on both metals. This residual charge compared well with the theoretical charge calculated from the capacitance of the two spheres separated by the cutoff distance for electron tunneling, with the potential difference equal to the work function difference of the metals. This experimental result confirms that the classic model of capacitors can also successfully describe effects at the nanoscale, further justifying the present model.

Having established the importance of the contact interface geometry for charge transfer, we conclude this section by analyzing how the transferred charge is distributed within the metal phases. For the majority of the studied particles (10 out of 15$), 90 \%$ or more of the total charge is retained directly at the contact interface; however, additional charging of the outer shell occurs in all nanoparticles as the shell is exposed to vacuum, although the sign of this charging is not the same in each system. Exceptions to this observation are all core-shell particles apart from the Janus particle with composition $\mathrm{Ag}_{346} \mathrm{Au}_{215}$.

Fig. 4 illustrates how the distribution of excess charge varies for core-shell nanoparticles with a gold core as the size of the core shrinks, while a similar analysis is given in Fig. S2 (ESI $\dagger$ ) for particles with a silver core. Clearly, the charge is not equally divided onto atoms within the same layer but accumulates onto the atoms which are coordinated to a greater number of foreign atoms. With only a single $\mathrm{Ag}$ shell layer, practically all of the transferred charge is contained at the interface. As the thickness of the shell grows, the outer shell becomes negatively charged as electrons from the next layer flow towards the Ag-vacuum interface. The magnitude of this charge is approximately constant for both particles. For nanoparticle $\mathrm{Ag}_{414} \mathrm{Au}_{147}$, this leaves the contact $\mathrm{Ag}$ 


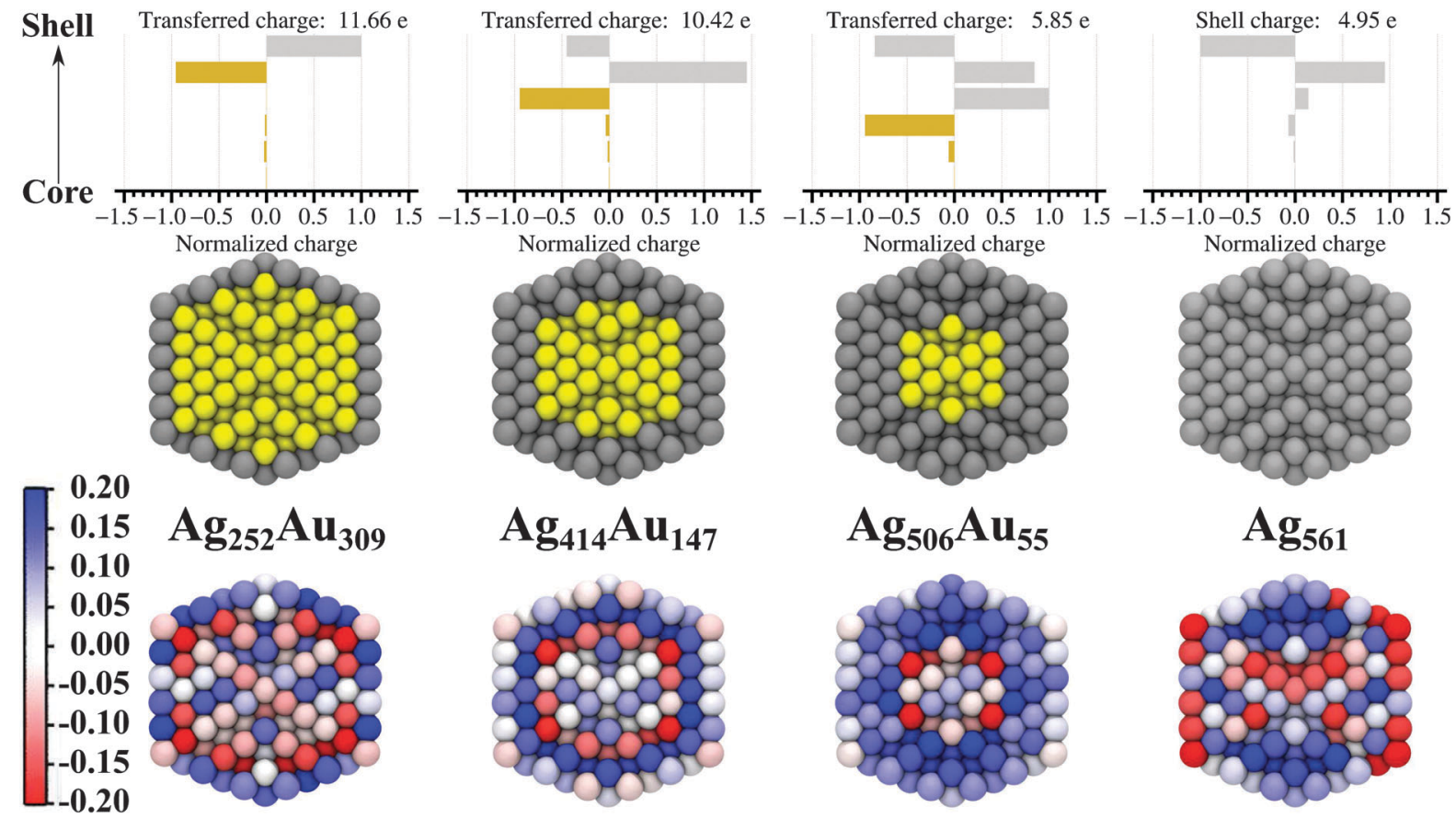

Fig. 4 Effect of core size on charge distribution in core-shell nanoparticles. At bottom, cross sections of the nanoparticles demonstrating the inhomogeneous distribution of charge onto individual atoms, with the actual atomic structure given for reference in the middle panel. At top, layer-bylayer net charge distributions. The charges have been normalized by the total amount of charge transferred from $\mathrm{Ag}$ to $\mathrm{Au}$, which is particle dependent. Negative charges indicate an electron excess. The limiting case of a pure Ag nanoparticle is included for reference, with charges normalized by the shell charge. Note, only a single atomic plane is visible in the cross sections, whereas all atoms are included in the layer-by-layer averaging.

layer at a greater net positive charge than would be expected solely from the magnitude of charge transferred to Au. The excess interfacial charging vanishes as the core further shrinks, resulting in a practically identical distribution of charge in noninterfacial layers when compared to the pure silver nanoparticle shown in the same figure. Overall, the trends for the nanoparticles with a silver core (Fig. S2, ESI $\dagger$ ) are similar to the results discussed here for particles with a gold core, but with the difference that the negative charge is spread over a greater number of $\mathrm{Au}$ layers than just the interfacial layer.

\subsection{Fermi level equilibration in bimetallic surfaces}

Charge transfer at the $\mathrm{Au} / \mathrm{Ag}$ interface was also investigated using fcc(111) metal slabs of varying thickness (both with and without periodicity), as shown in Fig. 5, with details of the computational methodology reported in the ESI. $\uparrow$ The distribution of charge in the pure metals was evaluated first, showing minor spill over of electrons into the surrounding vacuum at the surfaces. Layer-by-layer charge distributions for pure metal slabs are shown in the ESI $\dagger$ as a function of slab thickness, see Fig. S3. Upon contact between the two metals, some charge is transferred from silver to gold. Charge distributions for bimetallic systems composed of seven $(2 \times 2) \operatorname{Ag}(111)$ layers and one to four layers of $\mathrm{Au}(111)$ are given in Fig. 5.

Regardless of overlayer thickness, the addition of Au causes a reorganization of charge within the $\mathrm{Ag}$ slab resulting in charge transfer to Au which polarizes the interface. Fig. 5 shows that the effect is mainly contained to the first $\mathrm{Ag}$ layer closest to the interface, as the net charge of the remaining layers shows only minor deviations from pristine Ag values. Only a small fraction of the charge is present at the metal-vacuum interfaces, and cannot be accurately calculated.

The influence of surface morphology on charge transfer between $\mathrm{Ag} / \mathrm{Au}$ was investigated by modeling various different Au nanoislands on $(4 \times 4 \times 7) \mathrm{Ag}(111)$, as illustrated in Fig. 5 . For convenience, the islands are named by an $n$-tuple, where $n$ is the number of Au layers in the island and each element in the tuple denotes the number of $\mathrm{Au}$ atoms in the particular layer (counted away from the $\mathrm{Ag} / \mathrm{Au}$ interface). Thus, e.g. the notation $(3,1)$ denotes a 2-layer thick $\mathrm{Au}$ island with 3 atoms in the first and 1 atom in the second layer, respectively.

In Fig. 5, we have plotted the distribution of net charge in the studied $\mathrm{Au}$ nanoisland/Ag systems, while the total charge transferred from silver to gold is given in Fig. S4 (ESI $\dagger$ ) for each surface system. Overall, the magnitude of charge transferred to the $\mathrm{Au}$ islands is greater than with pristine $\mathrm{Au}$ overlayers. Normalizing by the number of atoms, the average charge transferred to island (3) is $-0.167 e$ per atom and to island (6) it is $-0.133 e$ per atom, whereas the corresponding value for the one layer thick $\mathrm{Au}$ overlayer was $-0.096 e$ per atom. This result suggests that the amount of charge transferred to $\mathrm{Au}$ increases as the size of the Au island relative to the surface area of the $\mathrm{Ag}$ slab decreases. Furthermore, the distribution of charge is not homogeneous within the first $\mathrm{Au}$ nanoisland layer; instead, the net charge on the edge atoms is approximately half of the charge on corner atoms, see Fig. S5 (ESI $\dagger$ ). 

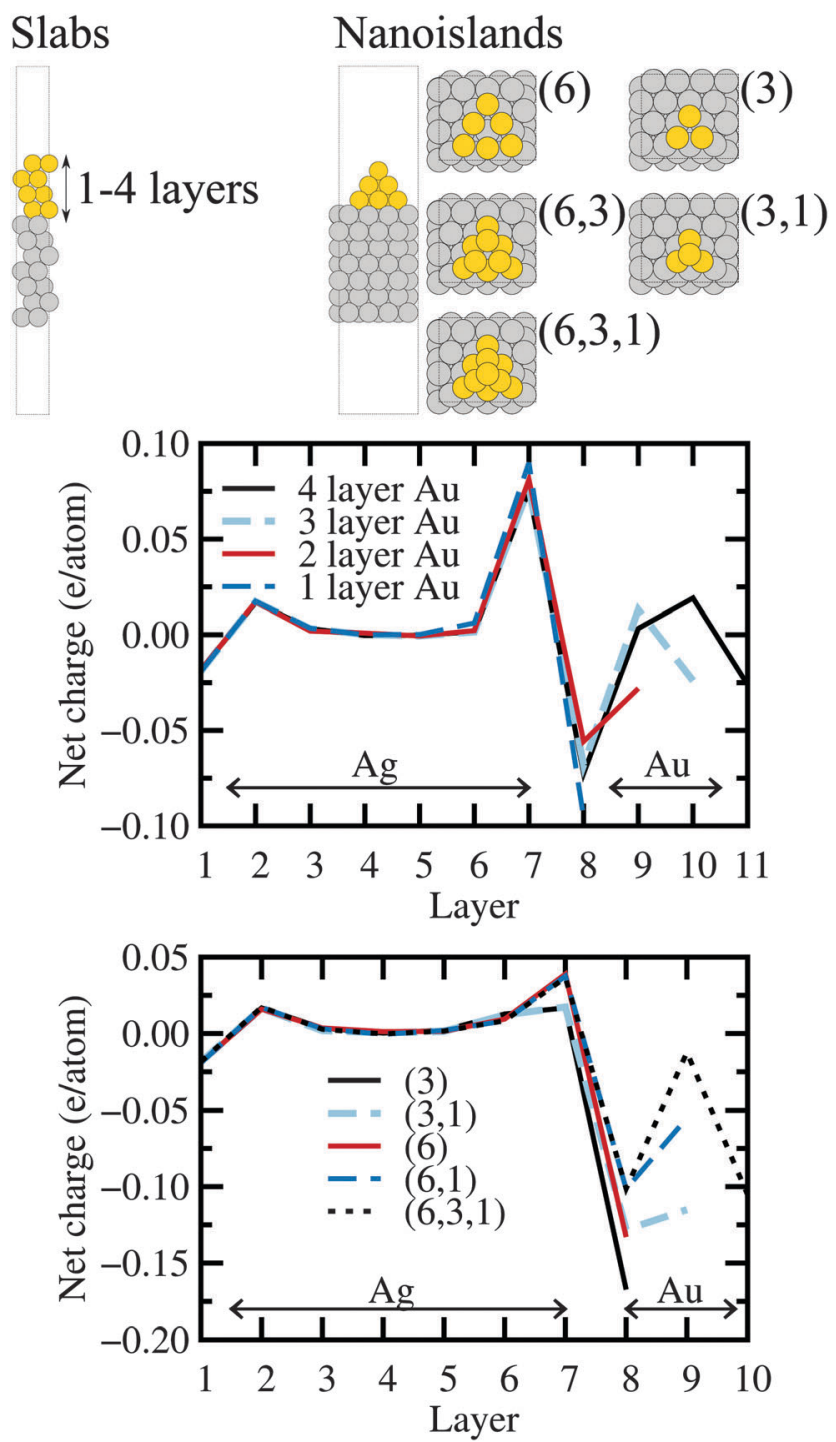

Fig. 5 Structures of the investigated $\mathrm{Au}$ islands on top of a $(4 \times 4 \times 7)$ $\mathrm{Ag}(111)$ slab and the combined system of seven $(2 \times 2) \mathrm{Ag}(111)$ layers in contact with $\mathrm{Au}$ (111) overlayers of different thickness (top). Top views of each island is shown in addition to a side view of the $(6,3,1)$-island. See main text for explanation on how the islands are named. Distribution of net charge in slab (middle) and nanoisland systems (bottom). Snapshots have been generated with ASE. ${ }^{41}$

If we now relate the computational capacitance of the surface systems to the geometrical structure of the $\mathrm{Ag} / \mathrm{Au}$ interface (see Table S2, ESI $\dagger$ ), similarly to the treatment for the nanoparticles, the correlation is very clear as demonstrated by Fig. 6 . This indicates that the amount of charge transferred depends significantly on the geometry, as was the case for the nanoparticles.

To understand the effects of periodicity, systems consisting of $7 \mathrm{Ag}(111)$ layers and 1-4 layers of $\mathrm{Au}(111)$, with four atoms in every layer, were also studied without periodic boundary conditions by placing the slabs in vacuum. Now, the atoms form rectangular cuboids, or square nanobars. In this case the environment of the atoms is markedly different, as different crystal facets on the sides of the nanobar (not on the $x y$ plane, as the (111) facet) become exposed to vacuum. The Fermi levels
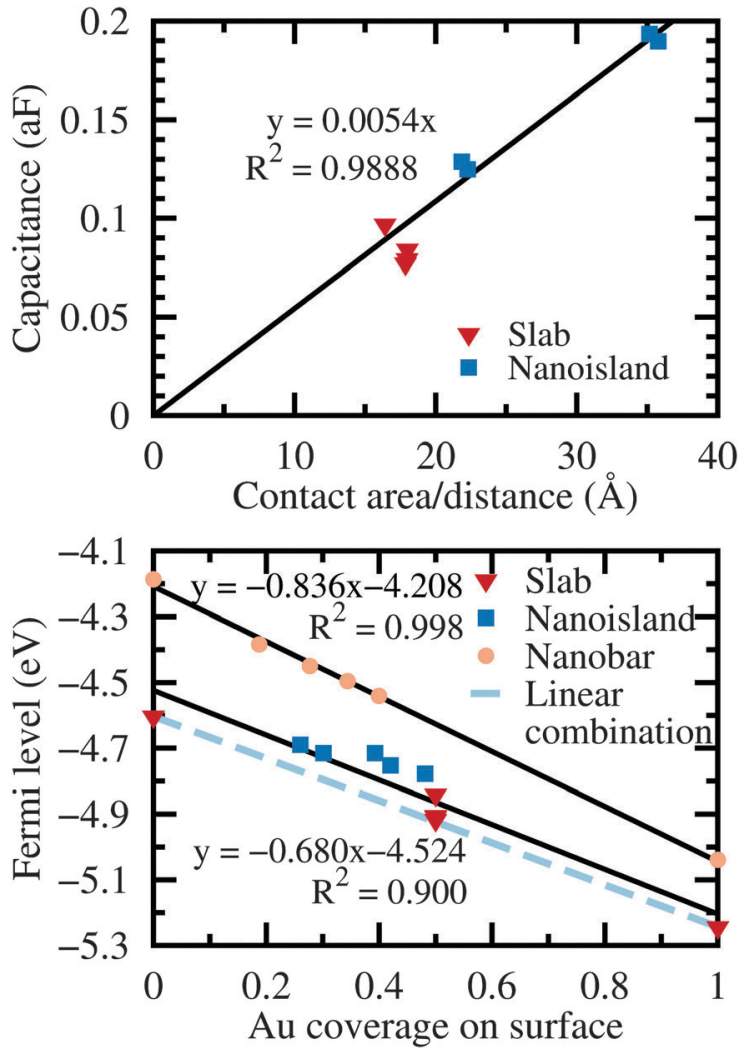

Fig. 6 Computational capacitance of the bimetallic surface systems as the function of contact area/contact distance (top). Fermi levels of bimetallic surfaces and nanobars as a function of Au surface coverage (bottom). Note that nanobars (pristine slab contacts calculated without periodic boundary conditions) have a different surface coverage of gold as the number of gold layers increases.

of all considered surface systems are shown in Fig. 6 as a function of outer layer gold coverage, which, for nanoislands and nanobars, was estimated from the surface area ratio of $\mathrm{Ag}$ and Au using the SASA method (see ESI $\dagger$ ).

Fig. 6 demonstrates that the Fermi level of nanobars can accurately be estimated with eqn (1), i.e., it is simply the coverage weighted average of the pure metal Fermi levels. The gold nanoisland surfaces, however, show slightly higher Fermi levels than predicted by eqn (1), while flat surfaces follow the theoretical curve well. This is probably because the electrostatic interactions between $\mathrm{Au}$ and $\mathrm{Ag}$ surfaces should be considered more carefully while calculating weighted averages of the Fermi level, as described by Sahni et al. ${ }^{35}$ The absolute values of the Fermi levels in Fig. 2 and 6 are not directly comparable since two different DFT methods were used in the calculations (see ESI $\dagger$ ), but they are of similar magnitude, and the slopes of the linear fits are very similar. These results further confirm that the Fermi level of a multimetallic structure is strongly dependent on the surface coverage. The calculations performed on the nanobars additionally confirm that the Fermi level of the system is also strongly dependent on which facets are exposed to the surrounding vacuum, as described earlier. ${ }^{35,36}$ The Fermi level of an infinite $\mathrm{Ag}(111)$ layer changes from $-4.60 \mathrm{eV}$ to $-4.19 \mathrm{eV}$ and the work function of $\mathrm{Au}(111)$ changes from 
$-5.24 \mathrm{eV}$ to $-5.04 \mathrm{eV}$ when periodic boundary conditions are removed and different crystal facets perpendicular to the (111)plane are exposed to vacuum. Furthermore, bimetallic contacts induce deformations into the crystal structures of both metals, even when the size of the metal atoms is as similar as gold and silver. ${ }^{3,42,43}$ This can affect both the chemical potential of electrons in the metal and the surface potential of the metal surface. ${ }^{3,42,43}$ Indeed, in Fig. 6, calculated Fermi levels are only shown for unrelaxed surface systems, where optimum cell constants are used for both $\mathrm{Au}$ and $\mathrm{Ag}$; but if the bimetallic surfaces are allowed to relax, the $R^{2}$ value of the linear fit decreases from 0.90 to 0.85 .

\subsection{Finite element simulations of the electrostatics of a bimetallic Janus particle}

To better understand the charge distribution and the electric field induced by contact electrification in Janus particles, the nanoparticle with composition $\mathrm{Ag}_{294} \mathrm{Au}_{267}$ was approximated as a sphere, and the electrostatics of this system were solved with COMSOL Multiphysics ${ }^{44}$ in 2D axial symmetry mode, as detailed in the ESI. $\dagger$ The separation between the two metals was adjusted so that the charge transferred matched the value (8.4 $e$ ) obtained by DFT calculations. The potential distribution of the system is shown in Fig. S7 (ESI $\dagger$ ) with streamlines for the electric field. The potential difference between the spheres is in this case equal to the difference of Fermi levels of the pure metal nanoparticles $(0.86 \mathrm{~V})$.

Charge distribution at the surfaces of the nanoparticle is shown in Fig. 7 demonstrating that most of the transferred charge, 93\%, is retained at the contact interface between $\mathrm{Ag}$ and $\mathrm{Au}$, with some focus points in the corners. Despite the relatively small charge located at the far end of the particles $\left(2.7 \times 10^{-4} e \AA^{-2}\right.$, arc length $34 \AA$ ), a surface charge density of this magnitude would be sufficient to induce a surface potential of $0.607 \mathrm{~V}$ on an equivalent sphere composed of a single metal. As the calculated potential is only $0.43 \mathrm{~V}$, some of the surface charge is screened by the opposite charge on the other side of the sphere. The electric field is strongest in the gap, and vanishes almost completely five particle radii away from the sphere. Of course, these calculations are simplifications of the system geometry, but they allow understanding the magnitudes of charges expected on the surface. In fact, inspection of the cumulative charge along the arc length shows excellent correspondence with atomic DFT charges, see comparison in Fig. 7. Here, the DFT arc length is defined according to the illustration in Fig. S8 $(\mathrm{ESI} \dagger)$ and it is slightly longer than the finite element quantity as the nonspherical shape of the nanoparticle is included in the definition. While the overall features are the same in both models, the finite element simulation is, by construction, unable to capture inhomogeneities of the charge distribution at the contact interface exemplified by Fig. S9 (ESI $\dagger$ ). Atomistic simulations also predict the outer surface of the Janus particle to be negatively charged, which implies that noninterfacial atoms must carry an opposite charge to reproduce the observed net charge difference between metals.

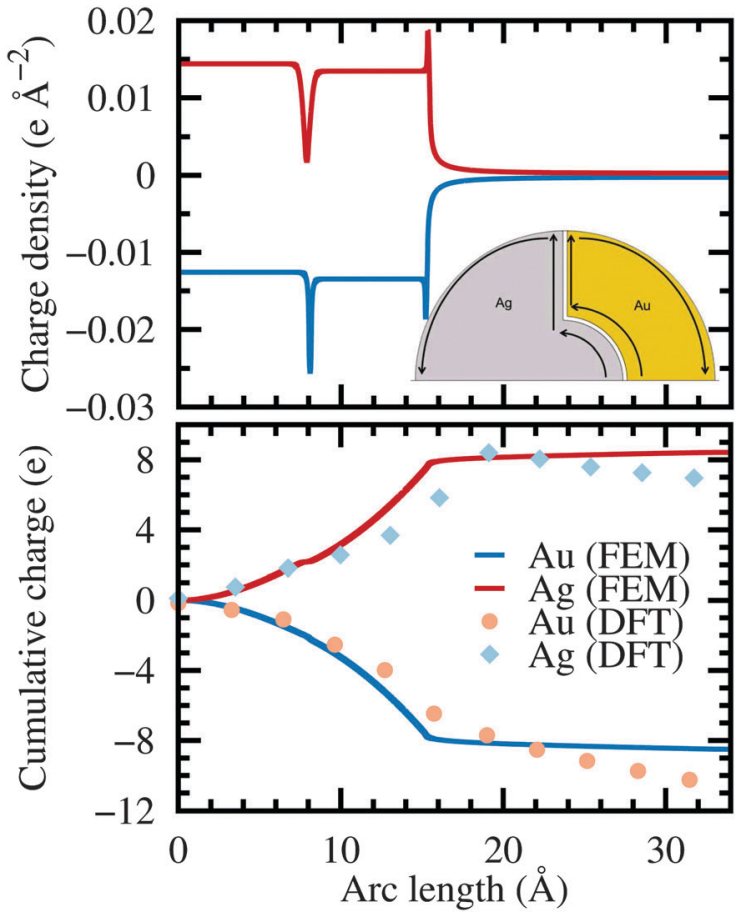

Fig. 7 Charge distribution at different surfaces of an $A_{29}{ }_{29} A_{267}$ Janus particle evaluated by a finite element simulation (FEM, top). The arc length starts from the gap at the center of the particle, as shown by arrows in the inset. Cumulative charge along the surfaces computed with DFT and FEM (bottom). Note, the arc length determined from DFT is slightly longer due to the nonspherical shape of the nanoparticle.

\section{Conclusions}

In summary, we have presented a new model to explain the redistribution of charge in bimetallic systems resulting from Fermi level equilibration after metal-metal contact, based on extensive density functional theory calculations for model nanoparticles containing nearly 600 atoms or $c a .1 .5 \mathrm{~nm}$ in diameter. In this model, the system is considered as a nanocapacitor formed between the metal phases with a potential difference equal to the work function difference of the pure metals, and where the magnitude of charge transferred is controlled by the contact interface geometry. This model is completely general, as verified by surface and continuum calculations; thus, it can be applied to understand charge redistribution in any multimetallic system regardless of size (going from nano- to macroscale) or shape, without the need for computationally intensive DFT calculations. However, as the capacitance model does not include the contribution of atomic scale charge transfer at the metal-vacuum interface nor the effects of atomic coordination, DFT calculations are still required for accurately describing these phenomena. Scaling relations between the equilibrium Fermi level and the metal surface coverage were also established.

We believe that the charge redistribution induced by Fermi level equilibration has a crucial role on, for example, the experimentally observed electrocatalytic, catalytic and optical properties of bimetallic nanosystems, and this work can help to 
explain the changes in these properties when compared to pure elements. Specifically, in bimetallic Janus particles, contact charging results in the formation of a permanent electrical dipole between metals, with most of the transferred charge located at the contact interface. Interfacial atoms on the outer surface of these particles are therefore intrinsically charged making them prospective active site candidates for catalysis applications. Lattice strain caused by atomic size mismatch is another phenomenon likely to affect catalytic properties and should considered in detail especially in particles where the size of the elements notably differ. ${ }^{45}$ On the other hand, the formation of an electrostatic dipole provides another tool to induce self-assembly in bimetallic Janus particles. Like Janus particles of a single metal covered with different surface groups, these bimetallic particles are expected to have very interesting self-assembly for formation of superstructures, which could have not been formed from the homogeneous particles.

\section{Acknowledgements}

P. P. acknowledges financial support from Fondazione Oronzio e Niccolò De Nora and EPFL. Prof. Hubert Girault (EPFL, Switzerland) is acknowledged for the numerous discussions and helpful comments during this work. This study was partly financed by the Academy of Finland through its Centres of Excellence Program, project no. 251748. N. H. is supported by Aalto University School of Chemical Technology through a PhD scholarship. Computational resources were provided by CSCthe Finnish IT Centre for Science.

\section{References}

1 V. R. Stamenkovic, B. Fowler, B. S. Mun, G. Wang, P. N. Ross, C. A. Lucas and N. M. Marković, Science, 2007, 315, 493-497.

2 X. Huang, Z. Zhao, L. Cao, Y. Chen, E. Zhu, Z. Lin, M. Li, A. Yan, A. Zettl, Y. M. Wang, X. Duan, T. Mueller and Y. Huang, Science, 2015, 348, 1230-1234.

3 Q. Jia, W. Liang, M. K. Bates, P. Mani, W. Lee and S. Mukerjee, ACS Nano, 2015, 9, 387-400.

4 R. Ferrando, J. Jellinek and R. L. Johnston, Chem. Rev., 2008, 108, 845-910.

5 P.-C. Chen, G. Liu, Y. Zhou, K. A. Brown, N. Chernyak, J. L. Hedrick, S. He, Z. Xie, Q.-Y. Lin, V. P. Dravid, S. A. O'Neill-Slawecki and C. A. Mirkin, J. Am. Chem. Soc., 2015, 137, 9167-9173.

6 F. F. Tao, Chem. Soc. Rev., 2012, 41, 7977-7979.

7 M. Sankar, N. Dimitratos, P. J. Miedziak, P. P. Wells, C. J. Kiely and G. J. Hutchings, Chem. Soc. Rev., 2012, 41, 8099-8139.

8 S. H. Hakim, C. Sener, A. C. Alba-Rubio, T. M. Gostanian, B. J. O'Neill, F. H. Ribeiro, J. T. Miller and J. A. Dumesic, J. Catal., 2015, 328, 75-90.

9 D. Alloyeau, C. Ricolleau, C. Mottet, T. Oikawa, C. Langlois, Y. Le Bouar, N. Braidy and A. Loiseau, Nat. Mater., 2009, 8, 940-946.
10 D. J. Sellmyer, B. Balamurugan, B. Das, P. Mukherjee, R. Skomski and G. C. Hadjipanayis, J. Appl. Phys., 2015, 117, 172609.

11 M. Kuisma, A. Sakko, T. P. Rossi, A. H. Larsen, J. Enkovaara, L. Lehtovaara and T. T. Rantala, Phys. Rev. B: Condens. Matter Mater. Phys., 2015, 91, 115431.

12 M. D. Scanlon, P. Peljo, M. A. Méndez, E. Smirnov and H. H. Girault, Chem. Sci., 2015, 6, 2705-2720.

13 R. Mitric, C. Bürgel, J. Burda, V. Bonacic-Koutecky and P. Fantucci, Eur. Phys. J. D, 2003, 24, 41-44.

14 J. Zhu, P. Cheng, N. Wang and S. Huang, Comput. Theor. Chem., 2015, 1071, 9-17.

15 V. Bonačić-Koutecký, J. Burda, R. Mitrić, M. Ge, G. Zampella and P. Fantucci, J. Chem. Phys., 2002, 117, 3120.

16 Z.-B. Ding, F. Wu, Y.-C. Wang and H. Jiang, J. Chem. Phys., 2015, 142, 214706.

17 Y. Zhang and T. Shao, J. Phys. D: Appl. Phys., 2013, 46, 235304.

18 K. Laasonen, E. Panizon, D. Bochicchio and R. Ferrando, J. Phys. Chem. C, 2013, 117, 26405-26413.

19 M. Harb, F. Rabilloud and D. Simon, Phys. Chem. Chem. Phys., 2010, 12, 4246-4254.

20 J. Zeng, C. Zhu, J. Tao, M. Jin, H. Zhang, Z.-Y. Li, Y. Zhu and Y. Xia, Angew. Chem., Int. Ed., 2012, 51, 2354-2358.

21 S. Chen, S. V. Jenkins, J. Tao, Y. Zhu and J. Chen, J. Phys. Chem. C, 2013, 117, 8924-8932.

22 A. J. Logsdail and R. L. Johnston, J. Phys. Chem. C, 2012, 116, 23616-23628.

23 L. Hong, A. Cacciuto, E. Luijten and S. Granick, Nano Lett., 2006, 6, 2510-2514.

24 A. Walther and A. H. E. Müller, Chem. Rev., 2013, 113, 5194-5261.

25 L. Wang, L. Xu, H. Kuang, C. Xu and N. A. Kotov, Acc. Chem. Res., 2012, 45, 1916-1926.

26 L. Xu, W. Ma, L. Wang, C. Xu, H. Kuang and N. A. Kotov, Chem. Soc. Rev., 2013, 42, 3114.

27 N. A. Kotov and P. S. Weiss, ACS Nano, 2014, 8, 3101-3103.

28 C. A. Silvera Batista, R. G. Larson and N. A. Kotov, Science, 2015, 350, 1242477.

29 S. Ye and R. L. Carroll, ACS Appl. Mater. Interfaces, 2010, 2, 616-620.

30 B. Lim, H. Kobayashi, T. Yu, J. Wang, M. J. Kim, Z.-Y. Li, M. Rycenga and Y. Xia, J. Am. Chem. Soc., 2010, 132, 2506-2507.

31 Q. Zhang, Y. H. Lee, I. Y. Phang, S. Pedireddy, W. W. Tjiu and X. Y. Ling, Langmuir, 2013, 29, 12844-12851.

32 W. Humphrey, A. Dalke and K. Schulten, J. Mol. Graphics, 1996, 14, 33-38.

33 J. Stone, MSc thesis, Computer Science Department, University of Missouri-Rolla, 1998.

34 J. P. Perdew, K. Burke and M. Ernzerhof, Phys. Rev. Lett., 1996, 77, 3865-3868.

35 V. Sahni, J. P. Perdew and J. Gruenebaum, Phys. Rev. B: Condens. Matter Mater. Phys., 1981, 23, 6512-6523.

36 C. Herring and M. H. Nichols, Rev. Mod. Phys., 1949, 21, 185-270. 
37 C. J. Fall, N. Binggeli and A. Baldereschi, Phys. Rev. B: Condens. Matter Mater. Phys., 2002, 66, 075405.

38 B. Lee and F. Richards, J. Mol. Biol., 1971, 55, 379-IN4.

39 A. Varshney, F. P. Brooks and W. V. Wright, IEEE Comput. Graph. Appl. Mag., 1994, 14, 19-25.

40 W. R. Harper, Proc. R. Soc. London, Ser. A, 1951, 205, 83-103.

41 S. R. Bahn and K. W. Jacobsen, Comput. Sci. Eng., 2002, 4, 56-66.
42 Q. Jia, C. U. Segre, D. Ramaker, K. Caldwell, M. Trahan and S. Mukerjee, Electrochim. Acta, 2013, 88, 604-613.

43 J. R. Kitchin, J. K. Nørskov, M. A. Barteau and J. G. Chen, Phys. Rev. Lett., 2004, 93, 156801.

44 COMSOL Multiphysics Modeling Software, COMSOL Inc., http://www.comsol.com/.

45 L. Li, F. Abild-Pedersen, J. Greeley and J. K. Nørskov, J. Phys. Chem. Lett., 2015, 6, 3797-3801. 